\title{
A Multilevel Product Model for Simulation-Based Design of Mechanical Systems
}

\author{
Kuang-Hua Chang* \\ School of Aerospace and Mechanical Engineering, The University of Oklahoma, 865 Asp Avenue,
}

Room 212, Norman, OK 73019

Kyung K. Choi

Department of Mechanical Engineening, The University of lowa, lowa City, IA 52242

Jeff Wang and Chung-Shin Tsai

Structural Dynamics Research Corporation, 2000 Eastman Drive, Mifford, $\mathrm{OH} 45150-2789$

Edwin Hardee

Center for Computer-Aided Design, College of Engineening, The University of lowa, lowa City, IA 52242

Received 17 March 1998; accepted in revised form 13 June 1998

\begin{abstract}
This paper presents a multilevel product model that supports Simulation-Based Design (SBD) of mechanical systems, from preliminary to detailed design stages. The primary goal of the SBD is to achieve product designs featuring better performance and greater durability and reliability through computer-based modeling, engineering analysis, and design trade-off. A Computer-Aided Design (CAD) model combined with engineering parameters and mathematical equations that simulate physical behavior of the mechanical system constitute its product model for SBD. For preliminary design, improvement of system performance, including dynamics and human factors, is usually the primary focus. A CAD model with reasonably accurate physical parameters, such as mass properties of major components or assemblies, is defined as the base definition of the product model for SBD. A number of simulation models are derived from the base definition to support simulation of the mechanical system performance. A parametric study can be conducted to search for design alternatives using dimension parameters created in the parameterized CAD model. The CAD model and base definition are then refined from the preliminary design stage to support intermediate designs. Intermediate designs will primarily focus on product subsystem performance. A product model is evolved by refining geometric representation of mechanical components in CAD, and expanding product assembly into parts and subassemblies for further engineering analysis. Component designs for performance, such as fatigue, mechanical reliability, and structural performance, as well as maintainability are the primary focus in the detailed design stage. A detailed product model evolved from that of the previous design is needed. In the detailed design stage, a systematic design trade-off method supports design improvement. A High Mobility Multi-Purpose Wheeled Vehicle (HMMMV) is employed to illustrate and demonstrate the proposed product model.
\end{abstract}

Key Words: simulation-based design, concurrent engineering, product model, modeling and simulation, computer-aided engineering.

\section{Introduction}

Simulation-Based Design (SBD) is the application of computationally intensive, high fidelity analysis algorithms to product computer models to obtain realistic product performance, without the need for construction and testing of actual physical prototypes of the product under consideration [1]. In the concurrent design methodology, simulation data can be employed to determine an optimal product design through iterative modification and analysis of product computer models. The SBD also supports exploring a substan-

\footnotetext{
*Author to whom correspondence should be addressed.
}

tially larger number of design options than is possible in the typically prolonged and costly traditional design-build-test product development cycle.

The objective of the research presented is to investigate and develop a multilevel product model that supports SBD of mechanical systems, from preliminary to detailed design stages. With the fast pace of industry moving toward bringing cheaper and better quality products to market in a shorter product development cycle time, the SBD method that employs advanced computer simulation and analysis technologies provides an effective means for practicing Concurrent Engineering. The SBD supports CE, in particular, the version defined by the U.S. National Research Council, "CE: a de- 
sign engineering environment in which computer-aided design technology is used to assess and improve the quality of a product, not only during the active design phase but through its entire life-cycle" $[2,3]$.

A number of approaches have been developed and applied to assess and improve product performance, such as Knowledge-Based Engineering (KBE) [4], DFX (design for $\mathrm{X}$-abilities, such as manufacturability, serviceability, produceability, assembly, etc.) [5,6], life-cycle design [7], etc. The SBD method does not supersede any of the approaches listed. Instead, SBD specialized in the performance assessment and improvement of complex, large-scale, computeintensive mechanical systems. In the SBD method, product development task and the cross-functional team are decomposed according to disciplines and expertise. Based on a centralized product model, simulation models can be derived and product performance can be simulated concurrently across disciplines. Product performance obtained from multidisciplinary simulations is brought together for review by the cross-functional team. Design parameters that significantly influence the product performance and quality are determined and defined in the product model. With such a design model definition, a systematic design trade-off can be conducted to improve product performance and quality with a minimum number of design iterations [8].

Recent developments in Computer-Aided Engineering (CAE) have witnessed dramatic improvements in accuracy, speed, and sophistication in discipline specific software applications. Computer-based simulation technologies are maturing to the point that simulation results are comparable to test results [9]. However, product modeling requirements peculiar to specific CAE capabilities, coupled with a need for seamless model evolution from preliminary to detailed product design, have arisen as substantial roadblocks to the use of these technologies to achieve multidisciplinary design optimization.

An intelligent, design-focused product model must be established to provide a unified product representation from which all analysis models are derived and to which design change is applied. A Computer-Aided Design (CAD) system provides the principal means to develop the product design in the SBD environment. A CAD model combined with engineering parameters and mathematical equations that simulate physical behavior of the mechanical system constitute its product model for SBD. $\mathrm{CAD}$ and simulation models evolve from preliminary to detailed design stages, by refining fidelity of these models for more accurate simulations and realistic product representation. Using a parametric CAD/CAE design modeling paradigm, a substantially enhanced degree of commonality is maintained between design and analysis models that permit rapid design change propagation. The product model, as a result, is scaleable to various fidelity to support design stages from preliminary to detailed, using tightly connected CAD and simulation models.

Instead of addressing broad issues involving Concurrent
Engineering, life-cycle design, design integration and design for manufacturing, this paper presents a multilevel product model that supports designs of mechanical systems from preliminary to detailed stages in a multidisciplinary design environment. The remainder of the paper is organized as follows. In Section 2, a Simulation-Based Design (SBD) for Concurrent Engineering (CE) environment is introduced briefly. In Section 3 the multilevel product model is discussed. In order to illustrate and demonstrate the model, a design problem involving the High Mobility Multipurpose Wheeled Vehicles (HMMWV) suspension system has been conducted and is described in Section 4. Finally, conclusions of this research are presented in Section 5.

\section{Simulation-Based Design (SBD) for Concurrent Engineering (CE) Environment}

The SBD environment developed at the Center for Computer-Aided Design (CCAD) at The University of Iowa [1], as shown in Figure 1, supports the development of large-scale mechanical systems, particularly wheeled vehicles, tracked vehicles, and heavy equipment. The environment comprises an infrastructure that integrates a suite of engineering workspaces and a number of $\mathrm{CAD} / \mathrm{CAE}$ tools. The workspaces include the Simulation And Visualization Environment (SAVE) [1], the Tracked Vehicle WorkSpace (TVWS) [1], the Durability and Reliability Analysis Workspace (DRAW) [1], the Design Sensitivity analysis and Optimization workspace (DSO) [10], and the Maintainability Analysis Workspace (MAW) [11]. These engineering workspaces and supporting CAD/CAE tools are the principal functional components of this environment which utilizes mechanical system modeling techniques to support a broad spectrum of engineering disciplines. In addition to these engineering workspaces, the Iowa Driving Simulator (IDS) [12] is being integrated into the environment to provide a customer-in-the-loop design capability.

In the design evaluation phase, a CAD-based product model of the mechanical system is first constructed and brought into the SBD environment as the base definition (detailed in Section 3.1). After the base definition is generated, engineers can create various simulation models from the base definition, including dynamics, structural, reliability, and maintainability. After simulation models are created, engineering workspaces can be used for simulations. Using simulation results along with a design model, defined by associating design parameters and performance measures to the mechanical system, the parametric study and design trade-off can be performed for design improvement.

Dynamics simulation enables prediction of system dynamics performance and loads that act on components of mechanical systems. In the SBD environment, dynamics simulation can be performed using either TVWS for tracked vehicles or SAVE for wheeled vehicles and heavy equipment. Also, IDS can be used to address issues such as vehicle safety and design, driver behavior, and assessment. 

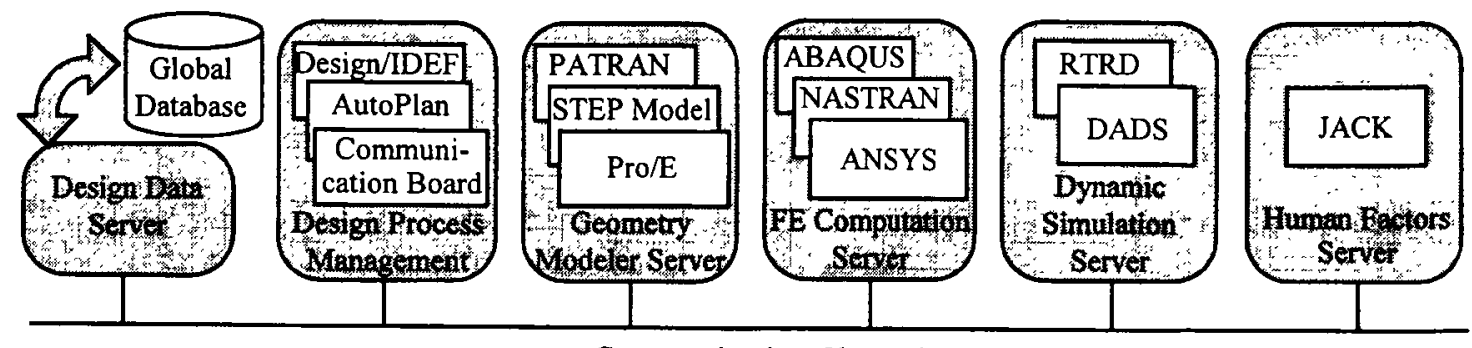

Communication Channel

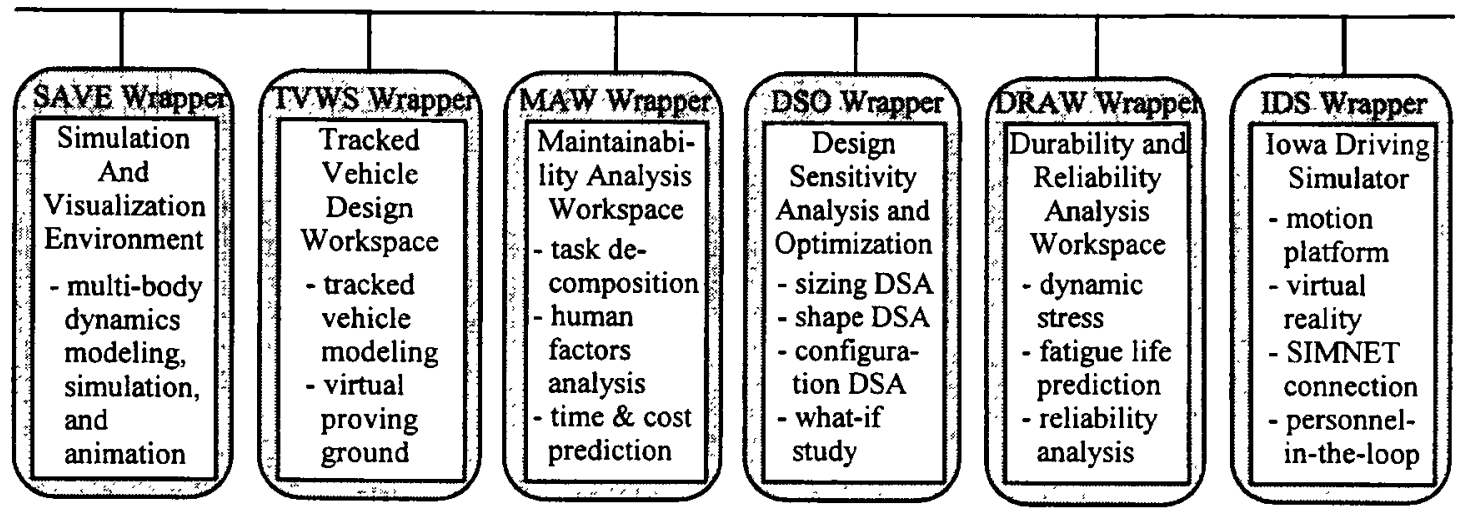

Figure 1. A simulation-based design environment.

Structural analysis provides the capability for predicting stresses, strains, displacements, natural frequencies, buckling load factors, and related structural performance, using structural geometric and material data and loads that are predicted by dynamics simulation or measured by experiment. In the SBD environment, engineers can use DSO to assess the structural performance of the components in the mechanical system, using finite element analysis (FEA).

The durability analysis capability provided in DRAW utilizes FEA and duty cycle data to compute dynamic stress of structural components or subsystems of the mechanical system. A local strain approach based on Morrow and SmithWatson-Topper methods for crack initiation fatigue life prediction, and a crack growth approach based on the modified Forman method for crack propagation fatigue life prediction have been implemented in DRAW.

DRAW also predicts the reliability of bearings, gears, and springs by combining dynamic loads and empirical data and equations published by manufacturers. Using techniques developed by the Anti-Friction Bearing Manufacturers Association (AFBMA) and data provided by bearing manufacturers in their bearing catalogs, the weighted $L 10$ value for each cycle can be calculated. Then, using the Palmgren-Miner linear damage summation rule, an L 10 value is calculated for the entire simulation period. The American Gear Manufacturers Association (AGMA) standard of gear life analysis and strength-stress distribution interference approach are employed for gear reliability analysis.

The maintainability analysis capability provided in MAW allows engineers to address design maintainability issues at various stages during the design process. MAW performs personnel/tool selection, task composition/animation, time analysis, and human factors analysis in support of rapid as- sessment of design maintainability. Maintainability design analysis in MAW employs computer models of maintenance personnel, mechanical systems design, support equipment, and simulation and animation of human-model interaction. The maintainability analysis incorporates the JACK anthropomorphic modeling tool, which was developed at the University of Pennsylvania.

Simulation results can be exported to the global database through workspace wrappers as well as posted to the Communication Board of the integration infrastructure of the SBD environment for use in defining a design model [8]. With the simulation results, engineers of the cross-functional team exchange design information effectively through the Communication Board, identify problematic areas in the mechanical system and define them as performance measures, and define design parameters from geometry dimensions in CAD models for the mechanical system. A parametric study or design trade-off is then conducted to vary design parameters for a desirable product performance, durability, and reliability.

The integration infrastructure consists of the following: (1) a Design Data Server (DDS) that manages the product definition of the mechanical system in the global database; (2) a set of tool servers, including a geometry modeling server, a finite element computation server, a dynamics simulation server, and a human factor server, that allow engineers to construct CAD and simulation models for simulations; (3) workspace wrappers that provide two-way data translation and transmission between workspaces and global database; (4) a process management utility that provides the chief engineer with capabilities to create and manage simulation design processes; and (5) the communication channel that distributes engineering workspaces, tools, and simulation data across a network of heterogeneous computers. 
In this paper, the focus is on product model. For a discussion of engineering capabilities and design applications of the SBD environment, the reader is referred to References [1] and [8].

\section{Product Model for SBD}

The primary goal of the SBD is to achieve product designs featuring better performance and greater durability and reliability through computer-based modeling, engineering analysis, and design trade-off. To support SBD, the product must be modeled at an adequate fidelity level so that the behavior of the mechanical system can be properly simulated. Product model in the SBD environment is centered around the base definition. To support multidisciplinary simulations, a base definition has to be established as the common ground among the cross-functional team. Base definition is composed of information retrieved from CAD models and non$\mathrm{CAD}$ parts and assemblies. Simulation models are derived from the base definition to conduct simulations and designs, as shown in Figure 2. Base definition is evolved from preliminary to detailed design stages by refining CAD models of the base definition, as illustrated in Figure 3. Simulation models derived from the base definitions also evolve among design stages. As a result, modeling effort is minimized. Product performance considered in the previous design stage needs to be revisited in the current design stage for confirmation of product performance using refined models. Also,

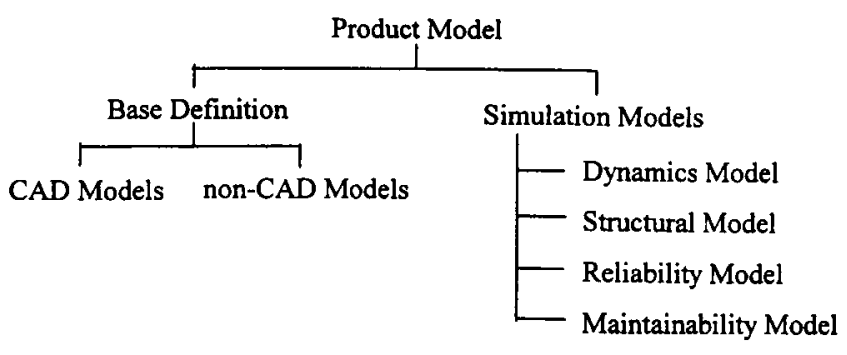

Figure 2. Product model definition.

product performance must be retained throughout design stages.

Note that the effort of creating product model in CAD environment can be substantial. Therefore, only interested parts and assemblies that will constitute simulation models need to be created in CAD. Parts and assemblies that do not involve design changes through geometric dimensions can be modeled as a set of physical parameters and mathematical equations, with their engineering behavior described without a $\mathrm{CAD}$ representation.

\subsection{Base Definitions}

Base definition contains two sets of data: entity hierarchy and entity attributes. The entity hierarchy describes how the components of the system are grouped together. The entity attributes for a part include mass, center of gravity, moments

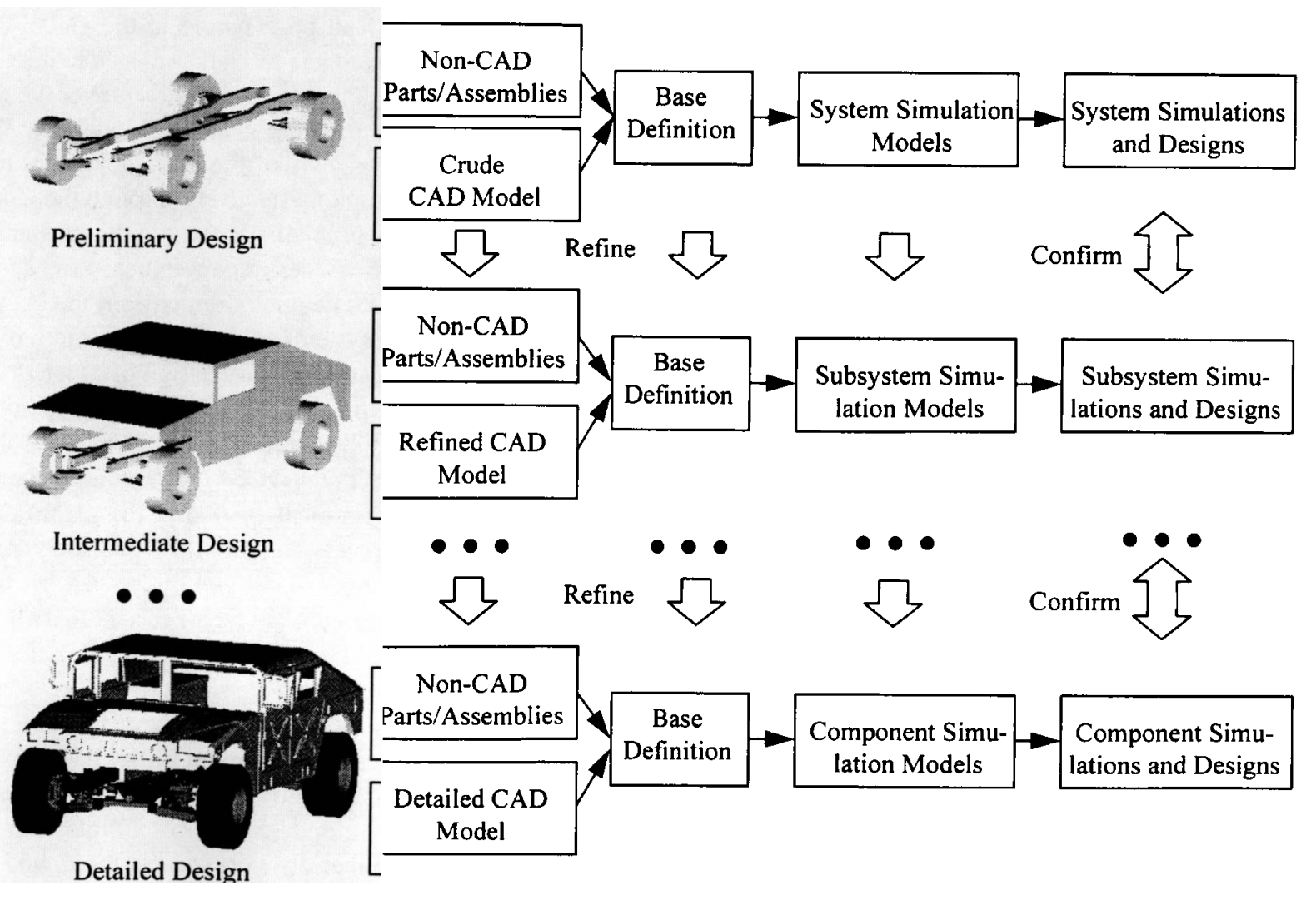

Figure 3. Multilevel product models for SBD. 
of inertia, material properties, and geometry information. Geometry information of the mechanical system is kept in the original $C A D$ format and later transferred to different formats to support various simulation model generations. In addition, parameters used to build the CAD geometry need to be extracted and later used as a base for parametric study and design trade-off.

Attribute information for an assembly differs from that for a part with the addition of assembly information describing the position and orientation of individual components relative to a local reference frame while no material properties are needed. Attributes of the product model can be browsed through a Web-based browser as shown in Figure 4.

A considerable portion of the mechanical system can have non-CAD representation, for example the engine, tire, and transmission of a ground vehicle. Characteristics of nonCAD parts and assemblies are usually described by engineering parameters and mathematical equations, such as engine angular velocity and horsepower. This non-CAD representation is added to the base definition as part of the product model.

In the SBD environment, design parameters are associated with dimensions of features in the parameterized CAD models. The design parameters are considered as part of entity attributes in the base definition. The feature-based design parameters serve as a basis to support parametric study and design trade-off across various engineering disciplines, where various performances of the mechanical system are simulated.

\subsection{Product Model for Preliminary Design}

During the preliminary design stage, performance of the mechanical system is usually unknown. It is desirable to compose a product model quickly, usually by employing legacy data or models, to assess its system performance. Also, design changes are needed to study the effect of changes in configuration, physical parameters, and geometric dimensions of the mechanical system to its performance.

The product model of preliminary design consists of base definition and simulation models, primary for dynamics simulation. The base definition is composed of a CAD model of the mechanical system with minimum parts and assemblies of crude geometry, and a set of engineering parameters

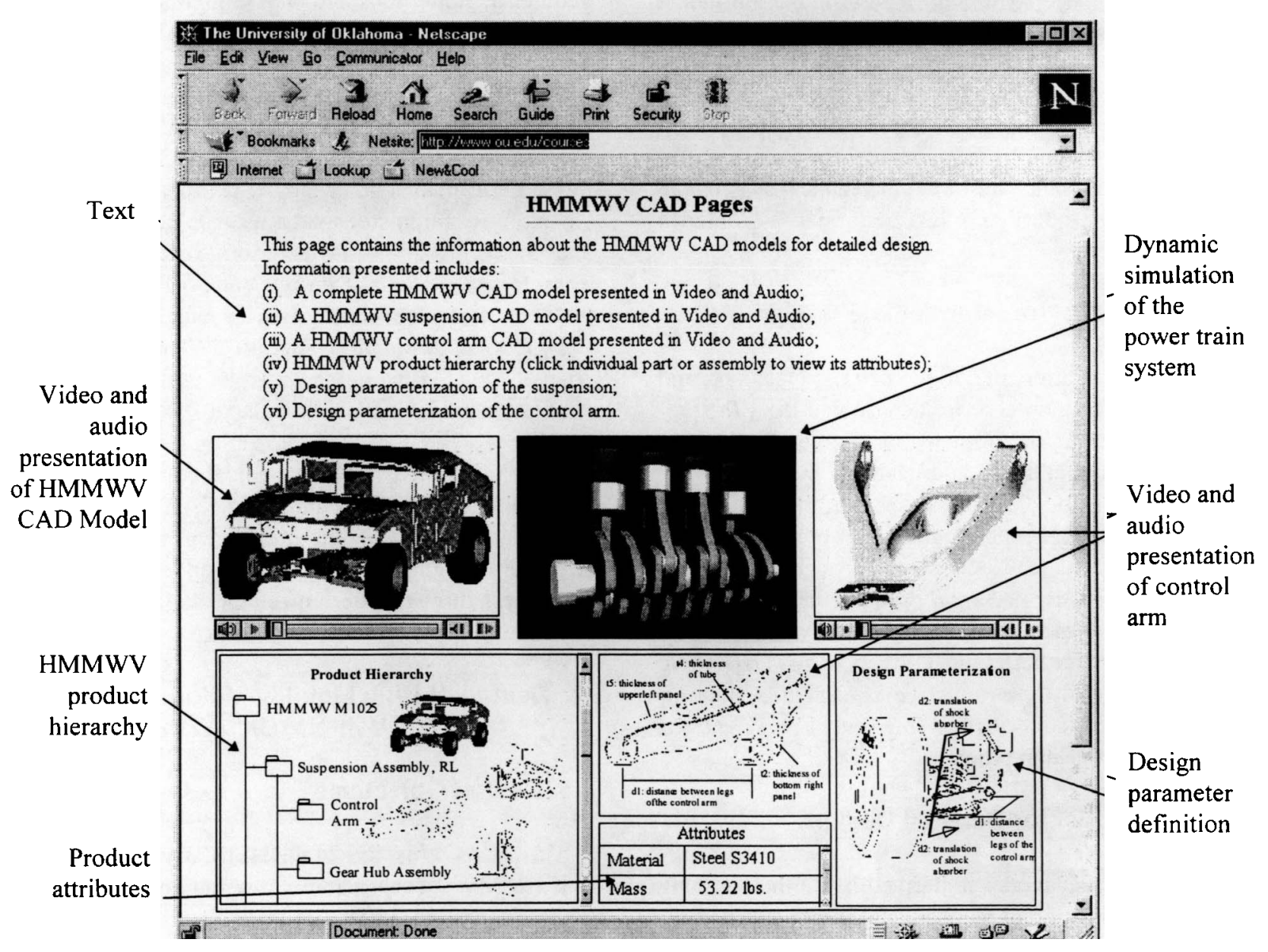

Figure 4. Web-based product browser. 


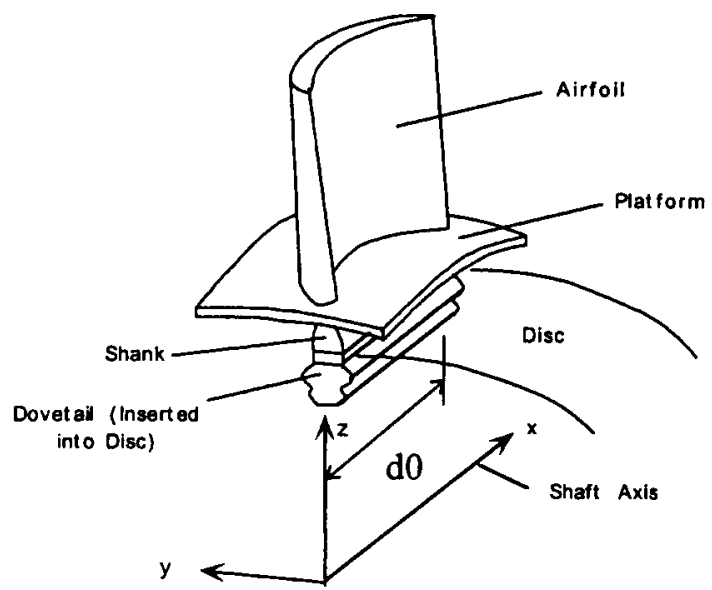

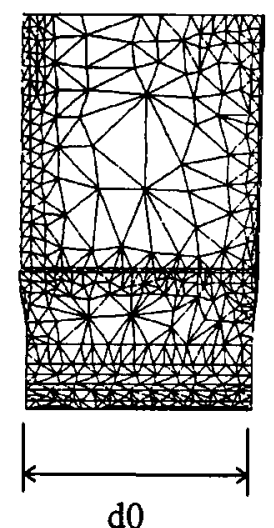

d0

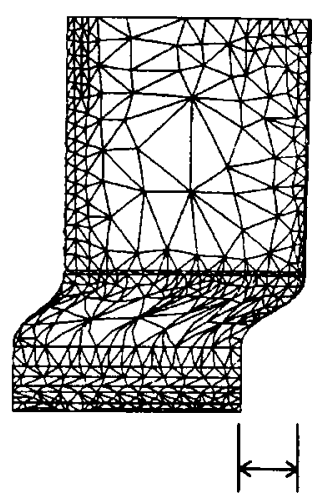

$\delta \mathrm{d} 0$

(a) Turbine Blade Physical Model

(b) Finite Element Model (c) Perturbed FE Model

Figure 5. Finite element model update using design velocity field.

and mathematical equations. Key geometric dimensions must be identified in the CAD model for design modification. A parametric study that perturbs parameters in the base definition to try out various design alternatives can be employed for the preliminary design stage. The parametric study is simple and easy to perform as long as a CAD/CAE mapping is established to support a fast simulation model generation. In this design stage, the $\mathrm{CAD} / \mathrm{CAE}$ mapping ties geometric dimensions in CAD to mass properties of a body and joint locations between bodies in the dynamics simulation models. The parametric study is possible for the preliminary design since the number of parameters to perturb is usually small. Dynamic behavior of the mechanical system and human factors are primarily product performance of interest for the preliminary design.

\subsection{Product Model for Intermediate Design}

Once the configuration, major geometric dimensions, and adequate physical parameters are determined from the preliminary design stage, performance of the mechanical subsystems are usually analyzed and designed next. A refined product model must be derived from that of the preliminary design to obtain a more accurate product representation and performance of mechanical subsystems through simulations. A product model is derived by refining product geometric representation and expanding product into assemblies or sub-assemblies for further engineering analysis. Depending on the complexity of the product being designed, none or more than one intermediate design stage may be encountered during the product design process.

\subsection{Product Model for Detailed Design}

A detailed product model is derived by further refining geometric representation of mechanical components and expanding product assembly into parts and sub-assemblies for further engineering analysis. Moreover, standard parts, such as screws, nuts, and bolts, are modeled accurately to support maintainability analysis of the mechanical system.

In a detailed design stage, component performance, such as structural fatigue, vibration frequency, buckling, and mechanical reliability are of primary interest. Therefore, more engineering disciplines are involved. Also, a large number of geometric dimensions and physical parameters, such as material properties, are present in the detailed product model; design change is much more sophisticated compared with that of the preliminary design stage. First, identification of a set of parameters that significantly affect performance of the mechanical components are not straightforward. Second, more performance in a single component is considered. Changing a parameter that improves component performance in one discipline may deteriorate performance in others. Therefore, a systematic design trade-off method employing mathematical algorithms, such as quadratic programming (QP) [13], is desirable to support design modifications [8]. Also, $\mathrm{CAD} / \mathrm{CAE}$ mappings must be established to facilitate simulation model regeneration due to design changes. At this design stage, the $\mathrm{CAD} / \mathrm{CAE}$ mapping ties geometric dimensions in CAD models of mechanical components to simulation models in various engineering disciplines. For example, for structural analysis, the mapping can be established between dimensions in a CAD model and structural finite element mesh through a design velocity field [14], as illustrated in Figure 5.

\section{Design of High Mobility Multipurpose Wheeled Vehicle (HMMWV)}

\subsection{Design Objectives}

The overall objective of the HMMWV design is to ensure that HMMWV suspension is durable and reliable after accommodating an additional armor loading of $2,900 \mathrm{lb}$. A multilevel design scenario will be presented in this section to illustrate and demonstrate the product model using the SBD 


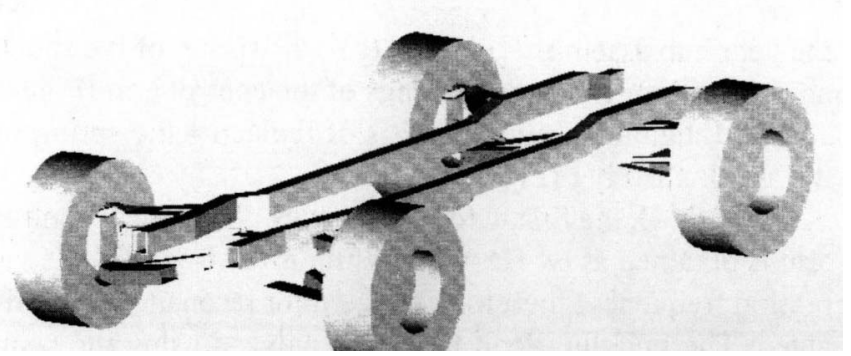

Figure 6. HMMWW CAD model for preliminary design.

environment. In the preliminary design stage, vehicle motion will be simulated and design changes will be performed to improve the vehicle gross motion. At this stage, dynamic behavior of the HMMWV suspension will be simulated and designed. The specific objective of the preliminary design is to avoid the problem of metal-to-metal contact in the shock absorber, and improve driver's comfort by reducing vertical acceleration at the HMMWV driver seat.

By modifying the spring constant to improve HMMWV suspension design at the preliminary design stage, the load path generated in HMMWV dynamics simulation is affected in the suspension unit. In the detailed design, the design objective is to assess and redesign durability, reliability, and structural performance of selected suspension components due to the added armor load and changes in load path. Also, maintainability of the suspension design will be assessed by performing a selected maintenance task.

\subsection{Two-Level Product Model}

In the particular case, a two-level product model is employed to support the HMMWV design example. In both levels, non-suspension parts, such as instrument panel, seats, lights, etc., are not modeled. Important vehicle components such as engine and transmission, are modeled using engineering parameters without depending on CAD representation. A low fidelity CAD model, consisting of eighteen parts, as shown in Figure 6, is created using Pro/ENGINEER [15] to support the preliminary design. This model has accurate joint definition, fairly accurate mass property, but less accurate geometry. The goal of the low fidelity model is to support vehicle dynamics simulation and human factors analysis. The low fidelity model is created using substantially less effort compared to that of the detailed model.

The detailed product model, consisting of more than 200 parts and assemblies shown in Figure 7 is created to support detailed design of suspension components. The detailed model is derived from the preliminary model by (i) breaking an entity into more parts and assemblies, e.g., the gear hub assembly shown in Figure 8, to simulate and design detailed parts, and (ii) refining geometry of mechanical components to support structural FEA, e.g., the lower control arm shown in Figure 9.

\subsection{Preliminary Design}

The HMMWV is assumed to be driven on the Aberdeen Proving Ground (APG4) test course with a constant speed of $20 \mathrm{MPH}$ for 23 seconds. A dynamics simulation model is derived from the base definition of HMMWV, as shown in Figure 10.

Using SAVE, severe metal-to-metal contact is identified within the shock absorber due to the added armor load and rough driving condition, as shown in Figure 11. The spring constant is adjusted to avoid any contact problem. The spring constant is increased in proportion to the mass increment of the added armor to maintain vehicle natural frequency. This design change not only eliminates the contact problem (see Figure 11), but reduces amplitude of vertical acceleration at the driver's seat that improves driving comfort (see Figure 12). However, the change alters the load path within the components of the suspension subsystem, e.g., shock absorber force acting on the control arm increases about $75 \%$, as shown in Figure 13.

\subsection{Detailed Design}

Simulations are carried out for fatigue, vibration, and buckling of the control arm (Figure 14); reliability of gears in

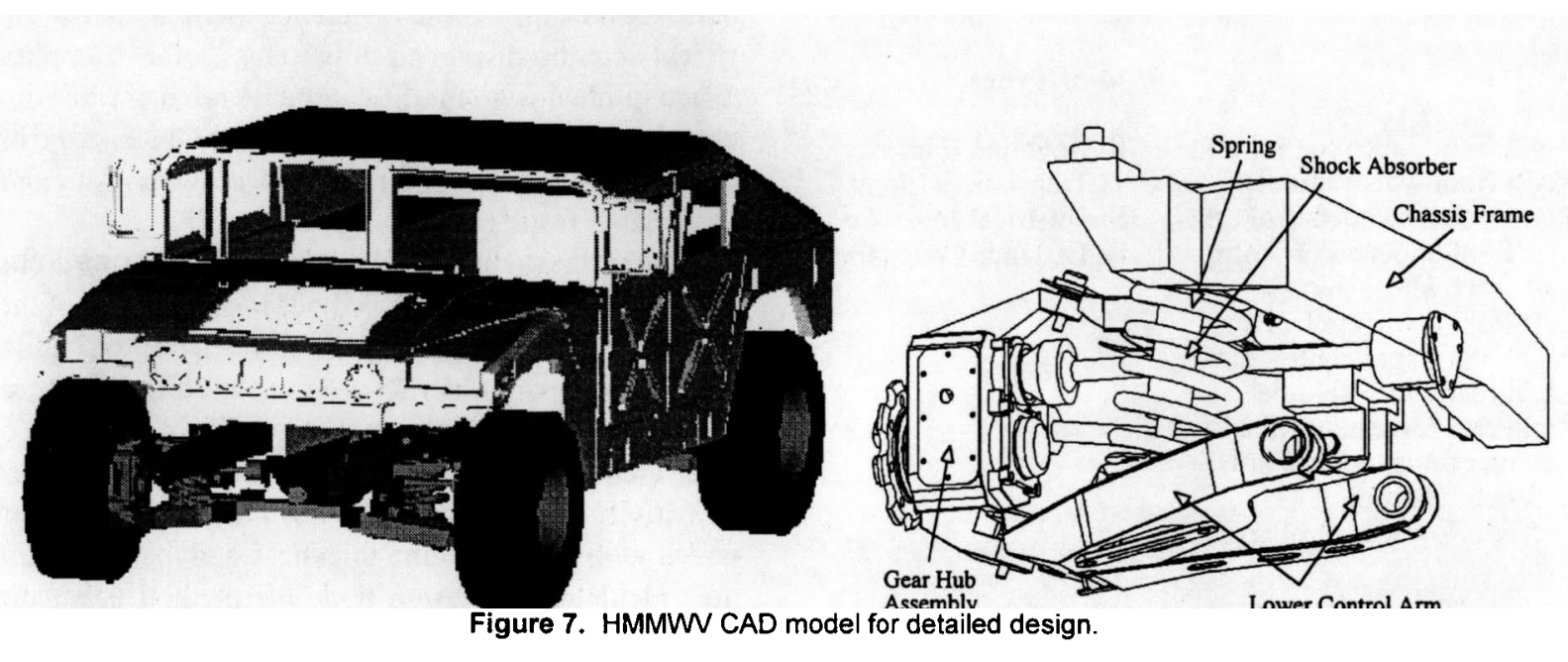



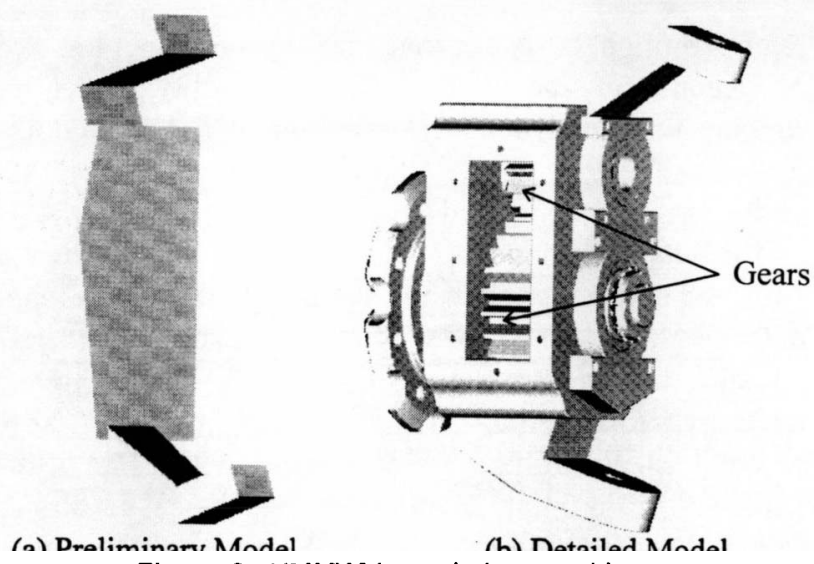

Figure 8. HMMWV gear hub assembly.
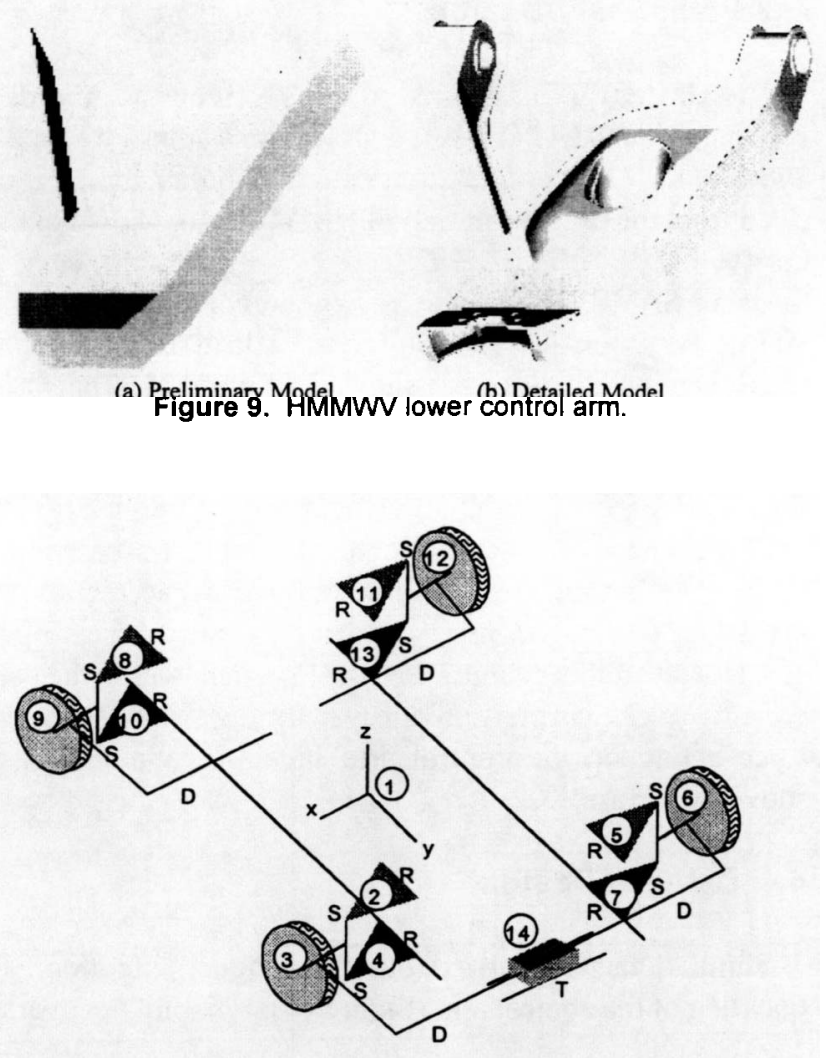

Body:

1 Chassis

2 Right front upper control arm

3 Right front wheel spindle

4 Right front lower control arm

5 Left front upper control arm

6 Left front wheel spindle

7 Left front lower control arm

8 Right rear upper control arm

9 Right rear wheel spindle

10 Right rear lower control arm

11 Left rear upper control arm

12 Left rear wheel spindle

13 Left rear lower control arm

14 Rack

Figure 10. HMMWN dynamics model. the gear hub assembly [Figure 8(b)], the spring of the shock absorber (Figure 7), and bearings of the control arm (Figure 13); and maintainability analysis of replacing the spring of the shock absorber (Figure 7).

Using DSO, the first natural frequency of the lower control arm is obtained as $64 \mathrm{~Hz}$, which is far away from vehicle vibratior. frequency; therefore, concern for resonance is eliminated. The buckling load factor is analyzed using the peak load at time 10.05 second of the 23 -second simulation period. The result shows that the control arm will not buckle even under the most severe load. Therefore, the current design is acceptable as far as buckling and resonance of the lower control arm are concerned.

Results obtained from the fatigue analyses using DRAW show that fatigue life (crack initiation) of the lower control arm degrades significantly, e.g., from $6.61 \mathrm{E}+09$ to $1.79 \mathrm{E}+$ 07 blocks (one block is 20 seconds) at critical areas (see Figure 15), due to the additional armor load and change of load path. Therefore, design must be changed to improved durability of the control arm. Reliability of bearing, gear, and spring at $99 \%$ fatigue failure rate is $2.18 \mathrm{E}+07,3.36 \mathrm{E}+06$, and $1.27 \mathrm{E}+02$ blocks, respectively. Fatigue life of the spring at the required reliability is not desirable.

\subsection{Design Trade-Off}

Eleven design parameters, including geometric dimensions (d1 and $\mathrm{d} 2$ in Figure 16), material property (cyclic strength coefficient $K^{\prime}$ of lower control arm), and thickness of the control arm sheet metal ( $t 1$ to 77 in Figure 16) are defined to support design modification.

A global design trade-off that involves changes of more than one component is conducted first. Geometric design parameters $\mathrm{d} 1$ and $\mathrm{d} 2$ are to be modified to reduce loads applied to the control arm, bearing, spring, and gears in the gear hub so that durability and reliability of these components can be improved. Changes of $\mathrm{d} 1$ and $\mathrm{d} 2$ affect not only the lower control arm but upper control arm and chassis frame. Sensitivity coefficients of loads at discretized time steps (total ten selected time steps) with respect to parameters $\mathrm{d} 1$ and $\mathrm{d} 2$ are calculated using a finite difference method. Sensitivity coefficients can be displayed in bar charts (for example, Figure 17) to guide design modifications. A what-if study is carried out with a design perturbation of 0.6 and 0.3 in. for $\mathrm{d} 1$ and $\mathrm{d} 2$, respectively, to obtain a reduction in loads. An example of the what-if results is shown in Figure 18.

A local design trade-off that involves design parameters of a single component is carried out for the design of the lower control arm. Thickness design parameters $t 1$ to $t 7$ and the material design parameter $K^{\prime}$ are to be modified to increase fatigue life of the control arm. Fatigue life at ten nodes of its finite element model in the critical area is measured. Sensitivity coefficients of control arm fatigue life at these nodes with respect to the thickness and material parameters are calculated. A design trade-off method using quadratic programming algorithm (QP) is employed due to the large 

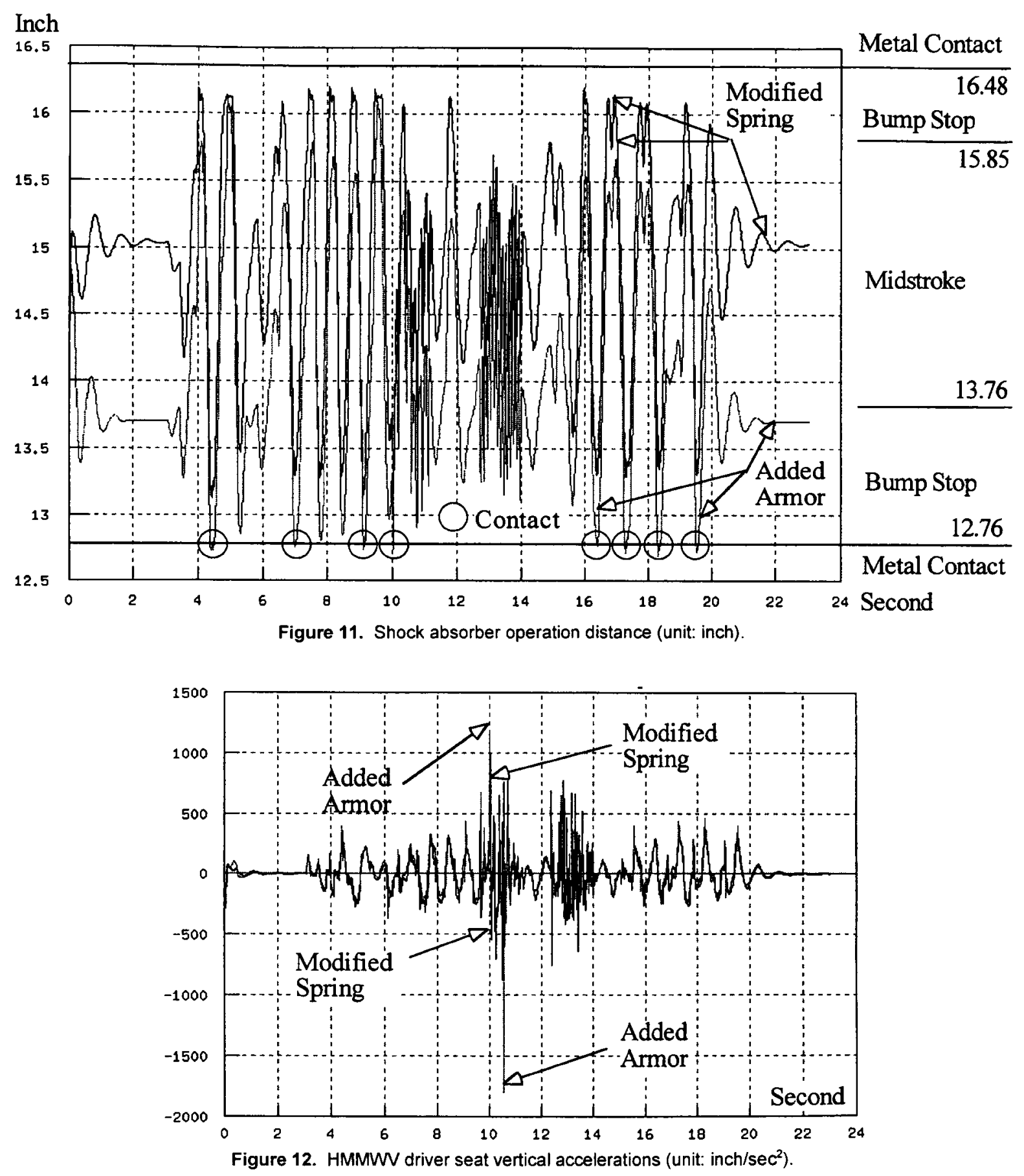

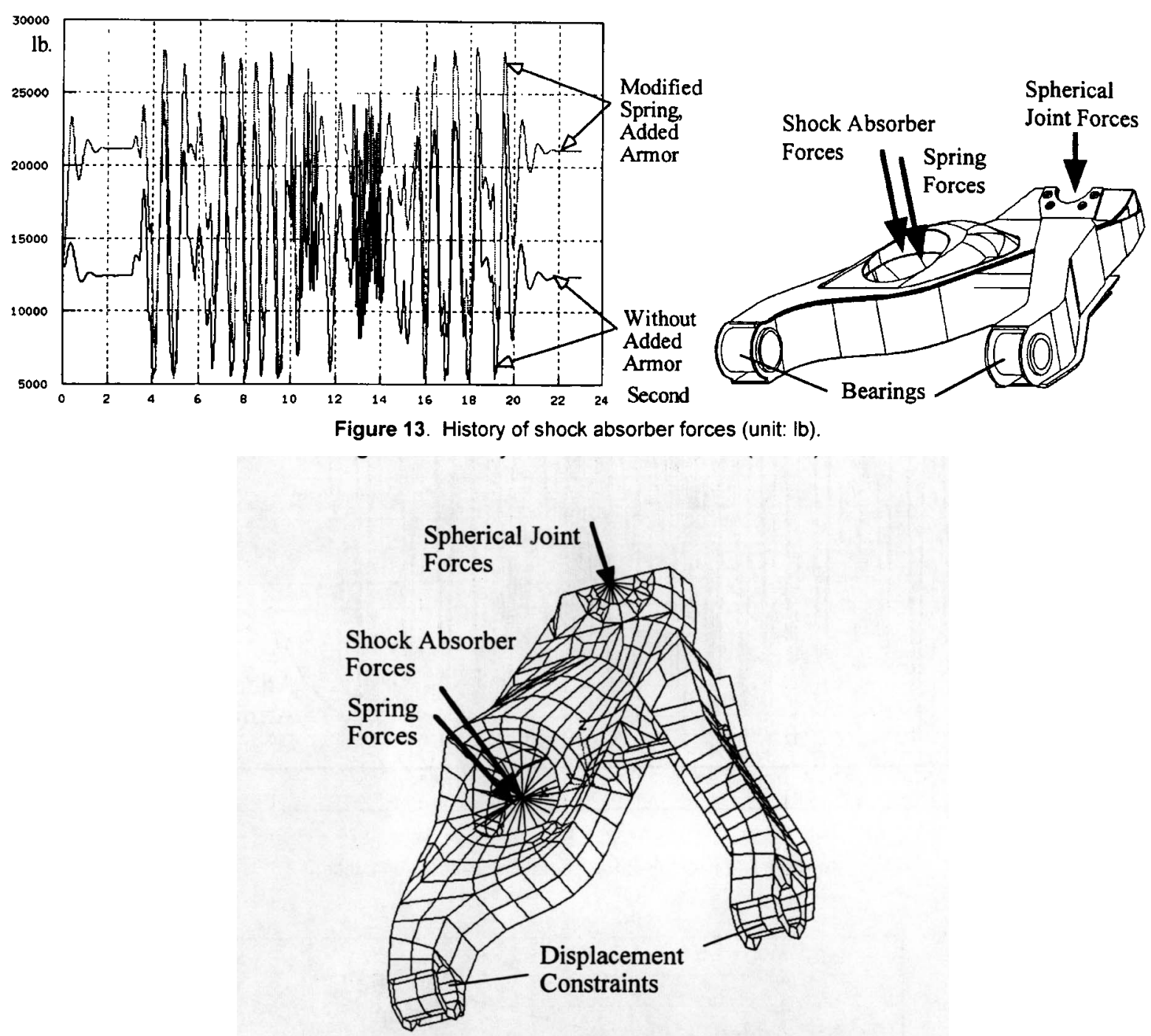

Figure 14. Lower control arm FE model.

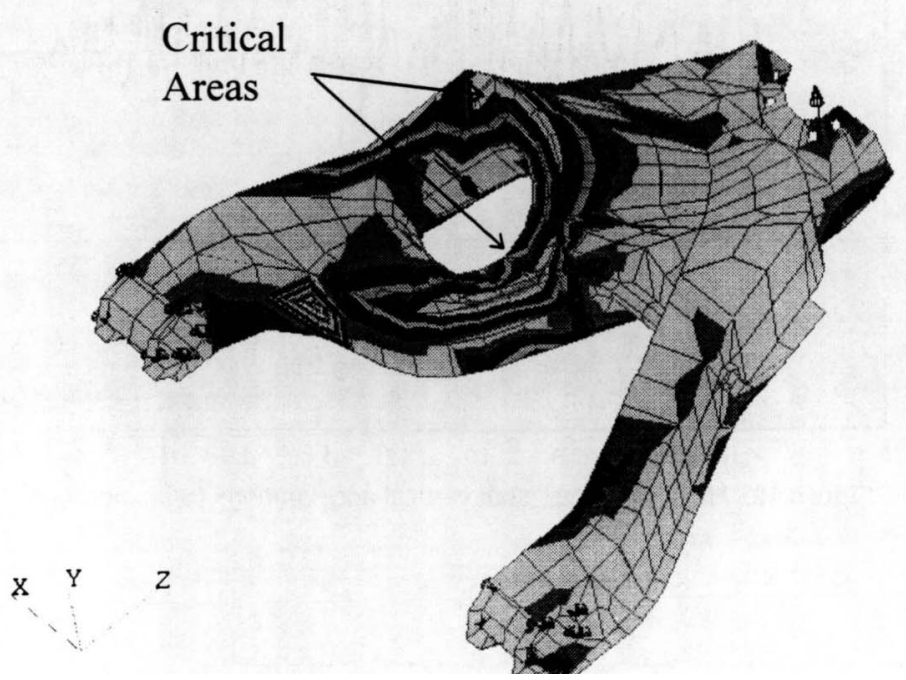

Figure 15. Fatigue life contour. 
d2: translation

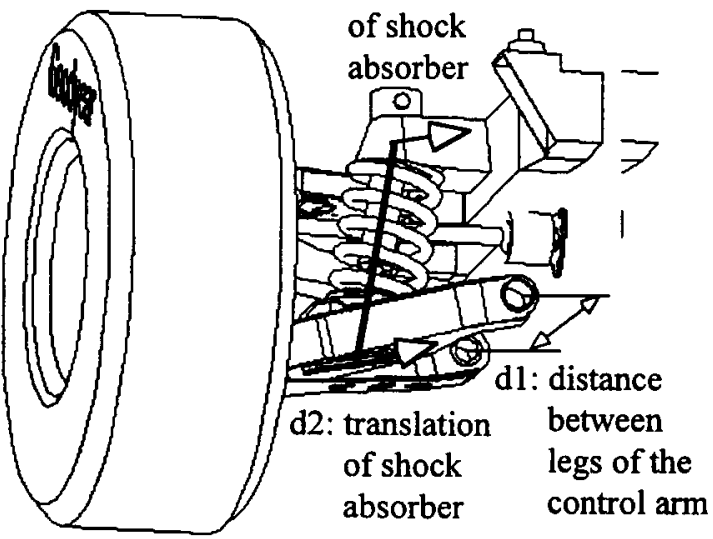

t5: thickness of

t7: thickness of ${ }^{t 5:}$ thickness of

t3: thickness of wall hole

d2: translation of shock absorber

t1: thickness of bottom left panel

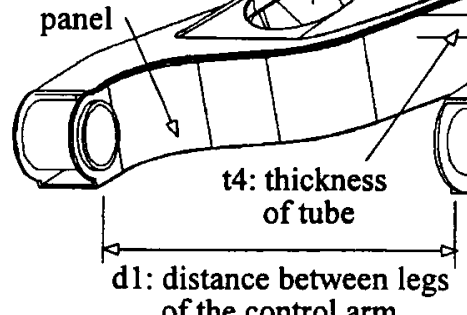
of the control arm semi-spherical panel

Figure 16. Design parameters defined for the control arm.

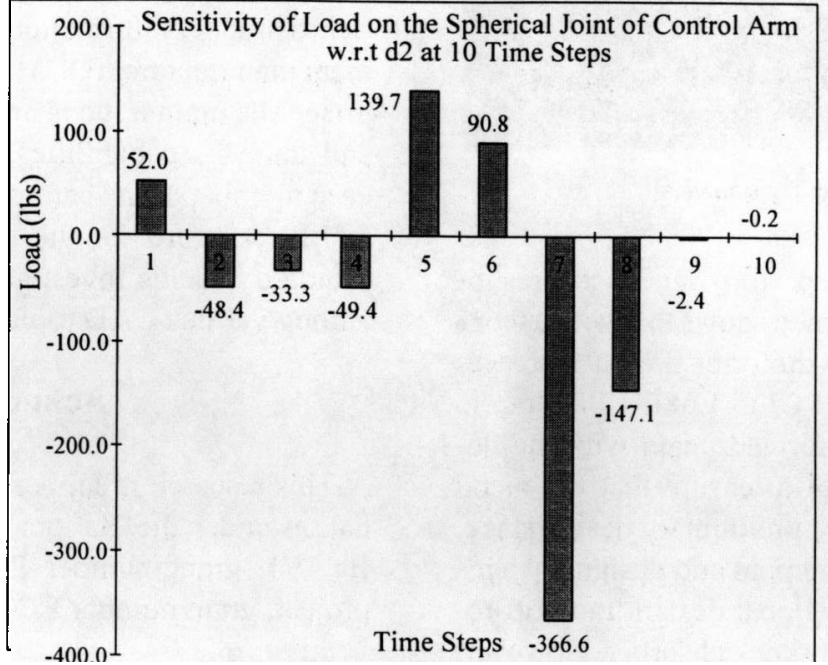

Figure 17. Bar chart of load sensitivity.

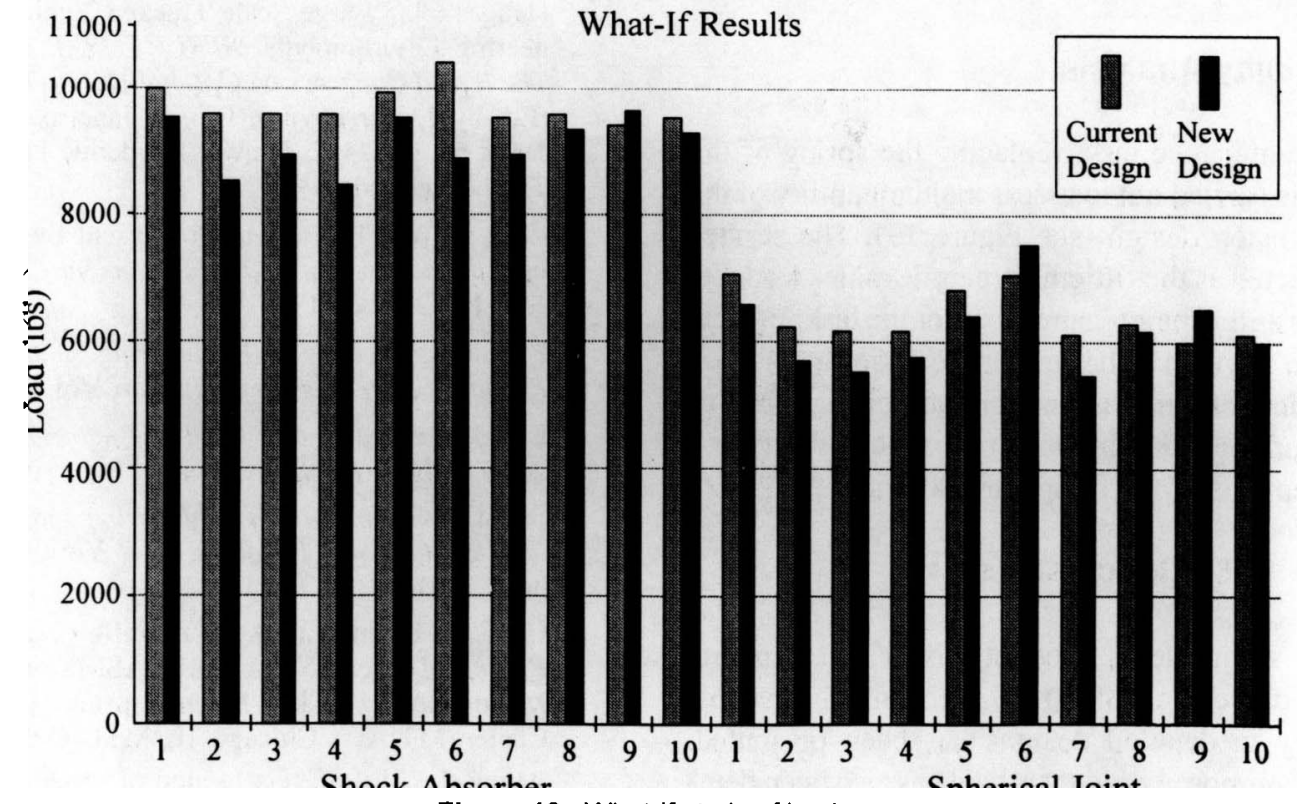

Figure 18. What-if study of loads 


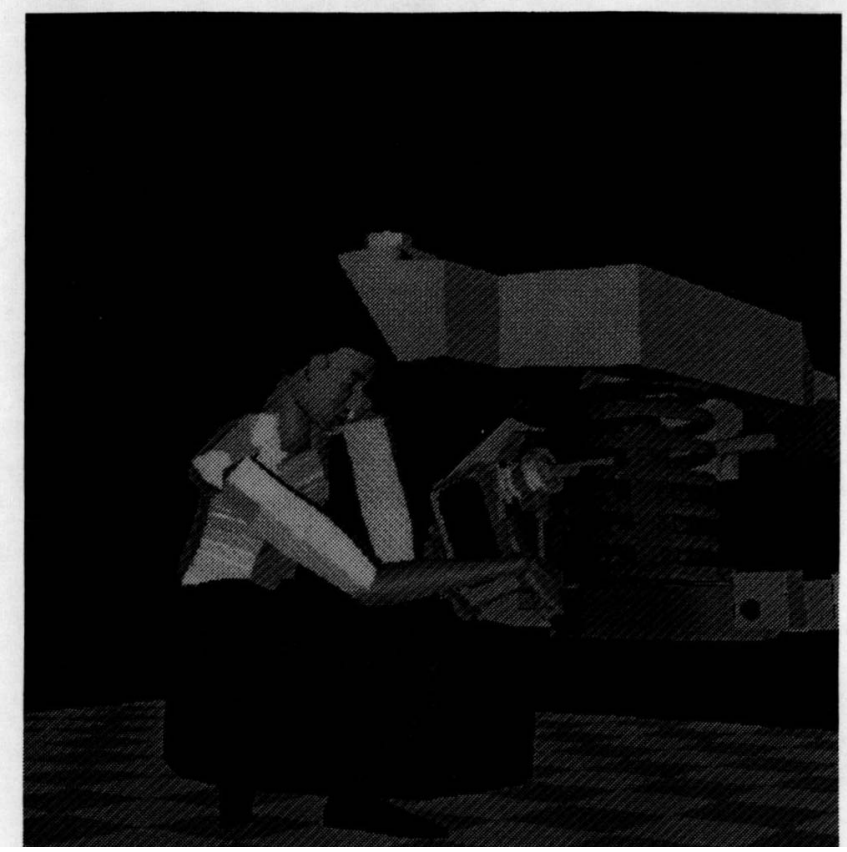

Figure 19. Maintainability analysis.

number of design parameters and performance measures involved. An improved design obtained shows that with a $0.6 \%$ weight increment, fatigue life at the critical area increases about ten times, i.e., from $1.79 \mathrm{E}+07$ to $1.68 \mathrm{E}+08$ blocks.

A dynamics simulation is performed again with the detailed model and modified design to ensure that the metal contact problem, considered in the preliminary design stage, does not occur due to model refinement and design changes in the detailed design stage. The global design trade-off reduces load applied to the spring of shock absorber. However, the reduction is not significant enough to increase spring fatigue life at the desired reliability. A maintainability analysis of replacing the spring is conducted and presented next.

\subsection{Maintainability Analysis}

A selected maintenance task, replacing the spring of the shock absorber, is carried out to assess maintainability of the HMMWV suspension design (see Figure 19). The service technician is selected as the fiftieth percentile male. A socket wrench is chosen as the maintenance tool for the task. Results of the simulation show that the time to perform the tasks is 132.5 seconds (time of removing major components, such as tire, are not included) and human strength, accessibility and visibility requirements of the design are satisfied.

\section{Conclusions}

In this paper, a multilevel product model that supports Simulation-Based Design (SBD) of mechanical systems, from preliminary to detailed designs has been presented. Feasibility of the proposed product model has also been demonstrated using an HMMWV example.
Several areas of the SBD environment related to product model are being refined and enhanced, including: (i) $\mathrm{CAD} / \mathrm{CAE}$ mappings to support a seamless and rapid simulation model regeneration for design iterations, (ii) graphicalbased product browser and editor to conveniently add design model definition, such as design parameters and performance measures, for parametric study and design trade-off, and (iii) extension of simulation capabilities, such as noise, vibration, and harshness (NVH), to support a broader spectrum of engineering disciplines.

In order to support an efficient design trade-off, design sensitivity analysis (DSA) using analytical methods for various mechanical performance with respect to a board range of design parameters, is essential. Currently, such capabilities are not available except for structural performance [16] and fatigue life [17].

Another research being carried out addresses a major barrier to product information integration among original equipment manufacturer (OEM) and its suppliers in a virtual enterprise. The main issue is on the typically incompatible design and analysis capabilities, particularly CAD systems, that each member of the enterprise employs in its operations. The STEP (standard for the exchange of product model data) standard is being investigated to support product exchanges among various $\mathrm{CAD}$ tools [18].

\section{Acknowledgement}

This research is supported by the National Science Foundation under the National Challenge program ENG-CISEIITA94, grant number ECS-9422722 and DARPA IPPD project, grant number EEC-9222784.

\section{References}

1. Choi, K. K., Wu, J. K., Chang, K. H., Tang, J., Wang, J., and Haug, E. J., "Large Scale Tracked Vehicle Concurrent Engineering Environment," STRUCTURAL OPTIMIZATION 93, The World Congress on Optimal Design of Structural Systems (Ed. by J. Herskovitz), Rio de Janeiro, Brazil, August 2-6, 1993, pp. 447-482, Kluwer Academic Publishers, Dordrecht, The Netherlands, 1995.

2. Ellis, L. W., "Practice in Concurrent Engineering," Proceedings of the International Management Conference, USA, pp. 15-118, October, 1992.

3. Prasad, B., Concurrent Engineering Fundamentals, Integrated Product and Process Organization, Vol. I, Prentice Hall, 1996.

4. Ertas, A. and Jones, J. C., The Engineering Design Process, 2nd Edition, John Wiley \& Sons, Inc., 1996.

5. Shina, S. G. Concurrent Engineering and Design for Manufacture of Electronic Products, New York, Van Nostrand Reinhold, 1991.

6. Chao, N. H. and Lu, S. C. Y. (ed.), Concurrent Product and Process Design, New Work, ASME, Computer Integrated Manufacturing (CIM)/Manufacturing Technology Analysis Center (MTIAC), Chicago, IL, MTIAC ST-88-02, 1988.

7. Alting, L., 'Life-Cycle Design of Products: A New Opportunity for Manufacturing Enterprises," Concurrent Engineering. 
Automation, Tools, and Techniques, Edited by Andrew Kusiak, John Wiley \& Sons, Inc., 1993.

8. Tsai, C. S., Chang, K. H., and Wang, J., "Integration Infrastructure for a Simulation-Based Design Environment," Proceedings of the Computers in Engineering Conference and the Engineering Data Symposium, ASME Design Theory and Methodology Conference, pp. 9-20, Boston, MA, August 1995.

9. Baek, W. K., and Stephens, R. I. "Fatigue Life Prediction and Experimental Verification for an Automotive Suspension Component Using Dynamic Simulation and Finite Element Analysis," Advances in Fatigue Lifetime Prediction Techniques, ASTM STP 1122, Mitchell, M. R., and Landgraf, R. W., Eds., pp. 354-368, Philadelphia, PA, 1992.

10. Chang, K. H., Choi, K. K., Tsai, C. S., Chen, C. J., Choi, B. S., and $\mathrm{Yu}, \mathrm{X}$. "Design Sensitivity Analysis and Optimization Tool (DSO) for Shape Design Applications," Computing Systems in Engineering, Vol. 6, No. 2, pp. 151-175, 1995.

11. Vujosevic, R., "Maintainability Analysis in Concurrent Engineering Mechanical Systems," Concurrent Engineering Research and Applications, Vol. 3, No. 1, 1995.

12. Kuhl, J. G., Papelis, Y. E., and Romano, R. A., "An Open Software Architecture for Operator-in-the-Loop Simulator Design and Integration," Concurrent Engineering: Tools and Technologies for Mechanical System Design, Springer-Verlag (ASI Series F), 1992

13. Arora, J. S., Introduction to Optimal Design, McGraw-Hill, 1989.

14. Hardee, E., Chang, K. H., Choi, K. K., Yu, X., and Grindeanu, I. "A CAD-Based Design Sensitivity Analysis and Optimization for Structural Shape Design Applications," The 6th AIAA/USAF/NASA/ISSMO Symposium on Multidisciplinary Analysis and Optimization, Paper No. 96-3990, pp. 77-87, Hyatt Regency Bellevue, Bellevue, WA, Sept. 4-6, 1996.

15. Parametric Technology Corporation, Release 15.0 Pro/ENGINEER Fundamentals, Parametric Technology Corporation, Waltham, MA, 1995.

16. Haug, E. J., Choi, K. K., and Komkov, V., Design Sensitivity Analysis of Structural Systems, Academic Press, New York, NY, 1986

17. Chang, K. H., Yu, X., and Choi, K. K. "Shape Design Sensitivity Analysis and Optimization for Structural Durability," International Journal of Numerical Methods in Engineering, Vol. 40, pp. 1719-1743, 1997.

18. Choi, K. K., Chang, K. H., Tsai, C. S., and Wang, J., "Information Integration for Simulation-Based Design," 1996 NSF Design and Manufacturing Grantees Conference, pp. 123-124, Albuquerque, NM, Jan. 3-5, 1996.

\section{Kuang-Hua Chang}

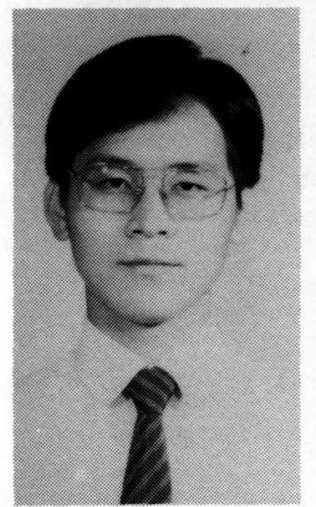

Dr. Chang is an Assistant Professor in the School of Aerospace and Mechanical Engineering (AME) at the University of Oklahoma (OU). He received a B.S. in Mechanical Engineering from Taipei Institute of Technology, Taiwan, in 1980; an M.S. and Ph.D. in Mechanical Engineering from the University of Iowa in 1987 and 1990, respectively. Since then, he joined the Center for Computer-Aided Design (CCAD) at Iowa as Research Scientist and CAE Technical Manager. In 1996, he joined Northern Illinois University as an Assistant Professor. In 1997, he joined OU.

His area of interests are Mechanical Fatigue and Reliability Analysis; Tools Integration for Concurrent Design and Manufacturing; Design Sensitivity Analysis and Optimization; and Internet-Based CAD/CAE/CAM using engineering multimedia, VRML and Java. Dr. Chang has published over 45 technical papers in these areas.

\section{Kyung K. Choi}

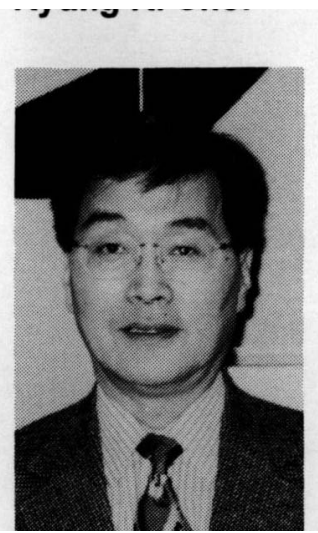

Dr. Choi is a professor in the department of Mechanical Engineering and Director of the Center for Computer-Aided Design. He received a B.S. in physics from YonSei University in Korea in 1970; an M.S. in mechanical engineering in 1977; and a Ph.D. in applied mathematics in 1980 , both from Iowa. Since then, he joined the Department of Mechanical Engineering at Iowa as an Assistant Professor (1984-1986), Associate Professor (1986-1990), and Professor (1990-).

His areas of interest are Mechanical System Analysis, Design Sensitivity Analysis, and Optimal Design; Computational Methods in Mechanics; Development of Design Sensitivity Analysis and Optimization Tools; and Development of Simulation-Based Concurrent Engineering Environment and CAE Integration.

\section{Chung-Shin Tsai}

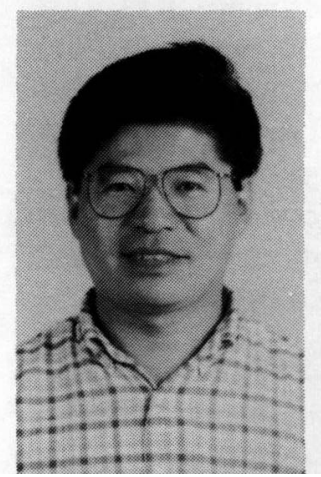

Dr. Chung-Shin Tsai is a senior software engineer at SDRC. He received a B.S. in Information Engineering and Computer Science from National Taiwan University in 1982; and a Ph.D. in Computer Science from the University of Iowa in 1992. He worked at the Center for Computer-Aided Design at the University of Iowa as a Research Scientist from 1992 to 1996 before he joined SDRC.

His areas of interest are Integrated Environment for Concurrent Engineering, Object-Oriented Programming Languages, Software Engineering, Internet-based Design Data Exchange, and $\mathrm{CAD} / \mathrm{CAE} / \mathrm{CAM}$ Integration. 


\section{Jia-Yi (Jeff) Wang}

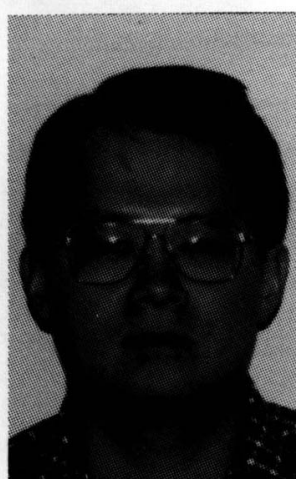

Dr. Jia-Yi Wang is a senior software engineer at Structural Dynamics Research Corporation. He received a B.S. in civil engineering from National Taiwan University in 1983; an M.S. in 1988; and a Ph.D. in mechanical engineering in 1992, both from the University of Iowa. He worked as an Associate Research Scientist at the Center for Computer-Aided Design from 1992 to 1996.

His areas of interest are Mechanism Design and Analysis, Design Sensitivity Analysis, Computational Methods in Multibody Dynamics, Variational Modeling, and CAD/CAE Integration for Concurrent Engineering.

\section{Edwin Hardee}

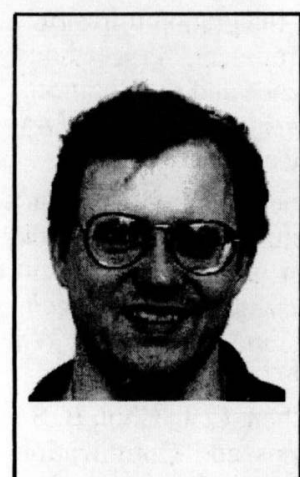

Dr. Hardee is a project leader at the Center for Computer-Aided Design in the department of Mechanical Engineering at the University of Iowa. He received a B.A. in philosophy and mathematics from Trinity College in Deerfield, Illinois in 1981 ; an M.S. in mathematics in 1987; an M.S. in computer science in 1993; and a Ph.D. in mathematics in 1993, all from the University of Iowa. Since 1994, he has been at the Center for Computer-Aided Design.

His areas of interest are software engineering for simulation-based concurrent engineering and integration of CAE tools. 